\title{
A global distribution of sporadic E events revealed by means of CHAMP-GPS occultations
}

\author{
M. Garcia-Fernandez and T. Tsuda \\ Research Institute for Sustainable Humanosphere, Kyoto University, Japan \\ (Received March 23, 2005; Revised June 22, 2005; Accepted August 10, 2005; Online published January 27, 2006)
}

\begin{abstract}
With the advent of the GPS/MET mission, the atmospheric sciences were given a new tool to enhance studies related to global and seasonal variations of different atmospheric parameters. In particular, it has proof valuable for the study of the ionospheric irregularities in the E layer, which before were performed basically on a local basis by ground based instruments. Using similar techniques applied to GPS/MET data to process the $50 \mathrm{~Hz}$ sampling rate GPS data to study the E layer electron density distribution, this work shows the global and seasonal distributions of the ionospheric irregularities of electron density, during high solar activity, by using the CHAMP satellite GPS data from January 1st 2002 to May 31st 2004. As pointed out by several theories on the E layer and previous studies with ground based data, the results presented here show that these irregularities have strong geographical and seasonal dependence, with enhancements in the auroral regions, in mid-latitudes and during the summer season.
\end{abstract}

Key words: Ionospheric irregularities, E layer, occultation, CHAMP.

\section{Introduction}

Since the proof-of-concept carried out by Low Earth Orbiters (LEO) such as the GPS/MET, the Abel inversion applied to occultation data has been proven to be a valuable tool to monitor different parameters of the ionosphere and the neutral atmosphere (Hardy et al., 1993; Hajj and Romans, 1998; Schreiner et al., 1999). In particular, the high sampling rate GPS data (at $50 \mathrm{~Hz}$ or $100 \mathrm{~Hz}$ ) gathered by LEOs has enhanced the research lines that involve neutral atmosphere as well as lower ionospheric layers (E layer). This is specially interesting towards the research of the coupling processes between the different layers of the atmosphere.

Before the advent of LEO occultations carrying GPS receivers for limb sounding, the ionospheric irregularities such as the sporadic E layer where basically studied with ground based instruments or rockets that provided with detailed but local information. With GPS-LEO occultations, global and seasonal variation of these irregularities can be studied and enhance the comprehension, for instance, of the sporadic-E layer and one of its causes explained with the generally accepted wind shear theory (Whitehead, 1961, 1989).

With the studies of ionospheric irregularities in the $\mathrm{E}$ layer performed during low solar activity period with the GPS/MET (Hocke and Tsuda, 2001a, b; Hocke et al., 2001, 2002), it could be seen the seasonal and geographic dependency of these irregularities. This work proposes a similar study but with the CHAMP data, during high solar activity.

Copyright (c) The Society of Geomagnetism and Earth, Planetary and Space Sciences (SGEPSS); The Seismological Society of Japan; The Volcanological Society of Japan; The Geodetic Society of Japan; The Japanese Society for Planetary Sciences; TERRAPUB

\section{Technique}

The technique used in this work is based on the one presented in Hocke et al. 2001a. The GPS phase observations of both frequencies $\left(L_{1}\right.$ and $\left.L_{2}\right)$ collected by the CHAMP satellite with sampling rate of $50 \mathrm{~Hz}$ are used to build the ionospheric combination $\left(L_{I}\right)$, which can be approximated as:

$$
L_{I}=L_{1}-L_{2} \simeq \alpha \cdot \int_{\mathrm{GPS}}^{R x} N_{e} \cdot d l+b
$$

where the integral of the electron density $\left(N_{e}\right)$ through the ray path is also known as the Slant Total Electron Content (STEC). Additionally to the STEC, the bias $b$ contains the instrumental delays and phase ambiguities, and is constant within the same observation arc. This feature will be used afterwards to remove it from the observable and to obtain the ionospheric fluctuations.

To obtain the height variation of the electron density fluctuations $(\Delta N e(h))$, the height of the tangent point for each ray is computed and associated to the corresponding $L_{I}$ observation. The bias can be eliminated afterwards by differentating with respect to height $\left(d L_{I} / d h\right)$. After removing the bias, the profile corresponds basically to the STEC variations, which can be approximated to the fluctuations of electron densities by multiplying by a constant factor. This constant factor corresponds to the lenght of the effective sounding volume. If one considers that the thickness of the spherically symmetric layer, where the electron density is assumed to be constant, is $0.6 \mathrm{~km}$, the length of this volune (a cylinder along the ray centered around the tangent point) can be approximated as $2 \sqrt{(} 2 r \Delta r)$, where $r$ is the Earth radius $(6370 \mathrm{~km})$ plus the height of the Elayer $(\simeq 100 \mathrm{~km})$, and $\Delta r$ is the layer thickness of $0.6 \mathrm{~km}$ (see more details in Ahmad and Tyler, 1998 and Hocke et al., 2001). Care must be taken with the fact that the CHAMP phase data shows 
large peaks with vertical periodicity of $1.5 \mathrm{~km}$ to $2 \mathrm{~km}$ approximately. To remove these peaks and in order to preserve the fluctuations with vertical scales of less than $7 \mathrm{~km}$, a 2$7 \mathrm{~km}$ bandpass filter has been applied to the fluctuation pro-

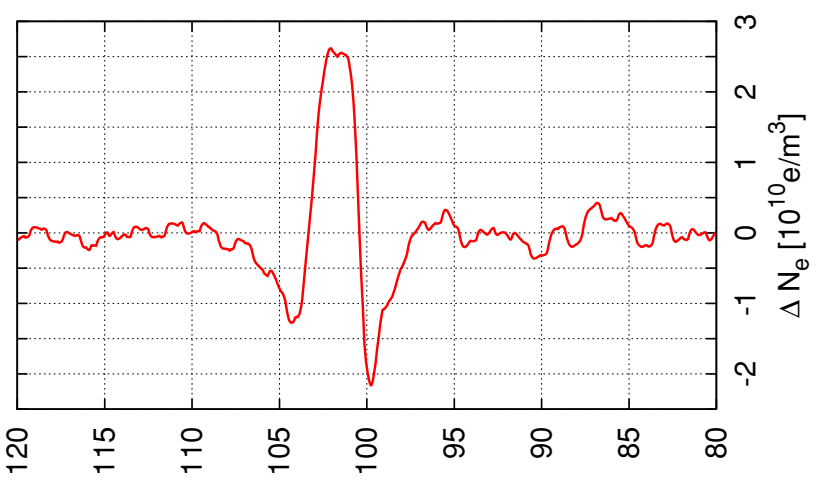

[யب्र]

Fig. 1. Examples of height fluctuations of electron density $\left(\Delta N_{e}(h)\right)$. This example corresponds to the CHAMP occultation with GPS satellite PRN17, the day April 10th 2003. The occultation took place at 116W20S, 3.8 hUT (20 LT). files. Figure 1 shows an example of fluctuations in height of electron density. The main characteristic of the morphology of these fluctuations is an enhancement around $100 \mathrm{~km}$ in the summer hemisphere. Although in some cases, the nightime profiles also show a certain enhancement, they are less important than during daytime. The ripples are due to the above mentioned peaks. Although a small remnant is still present, these peaks can be largely removed with the $2 \mathrm{~km}$ low pass filter applied to the profiles.

\section{Irregularities in the $\mathbf{E}$ Layer}

Using the technique explained in the previous section and the $50 \mathrm{~Hz}$ phase observations gathered by the CHAMP satellite during the period January 1st 2002 to May 31st 2004 , the vertical profiles of electron density fluctuations $\left(\Delta N_{e}(h)\right)$ of the E layer $(80 \mathrm{~km}$ to $130 \mathrm{~km})$ have been obtained. A total number of approximately 175.500 profiles were computed, which corresponds to an average number of 222 profiles per day. According to several theories of sporadic E formation such as the generally accepted windshear (Whitehead, 1961, 1989), the coupling between neutral winds in the atmosphere and the lower layers of the ionosphere is reflected in enhancements in the electron den-
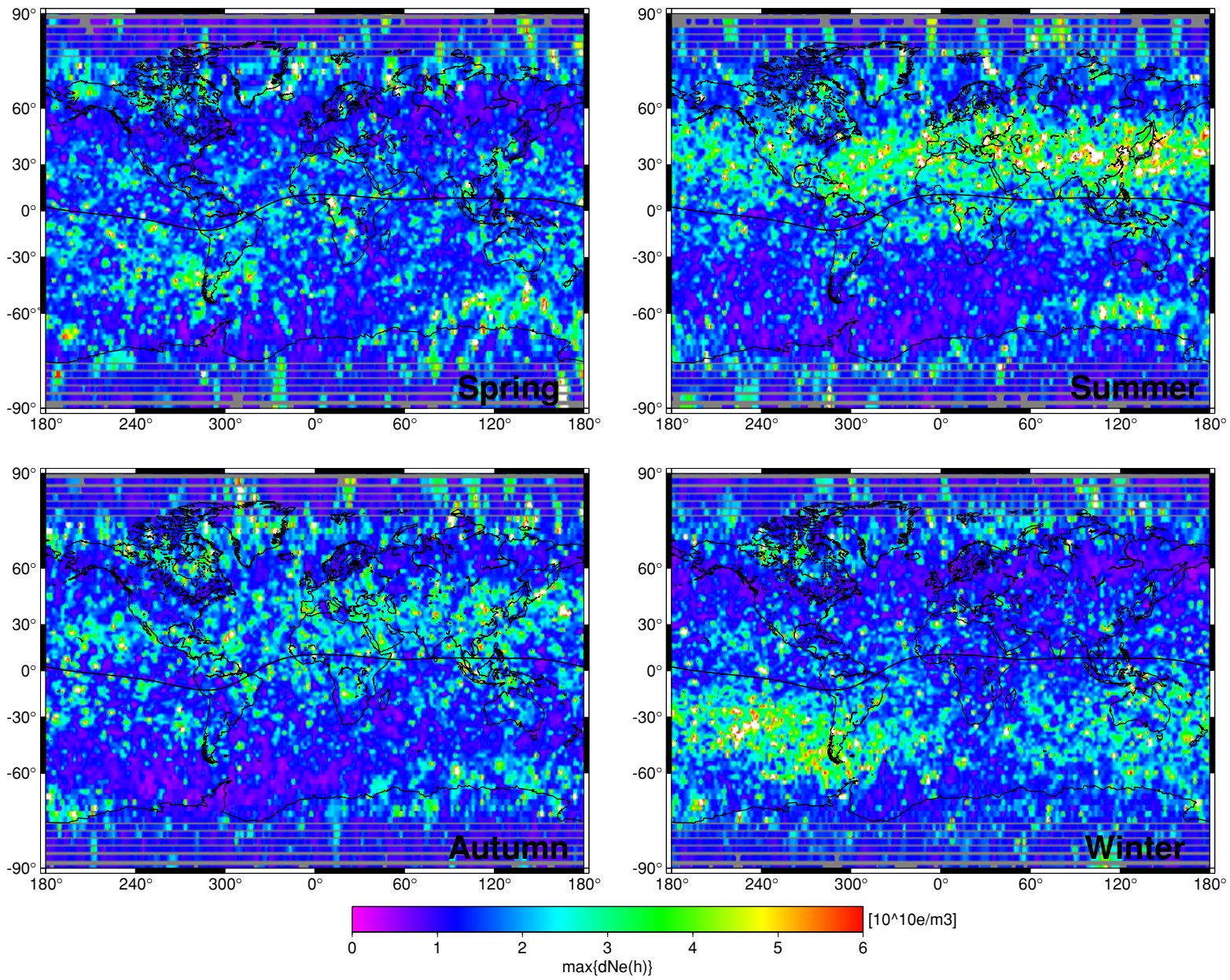

Fig. 2. World and seasonal distribution of ionospheric irregularities in the E region, corresponding to daytime period (5 LT to 22 LT). Periods shown are centered at the solstices or equinoxes, therefore Spring: day of year (doy) 046 to 126, Summer: from doy 127 to 218 , Autumn: from doy 219 to 310 and Winter: from doy 311 to 045 . GPS occultation data of CHAMP satellite from January 1 st 2002 to May 31 st 2004 have been processed. The middle black line in the map corresponds to the geomagnetic equator. 

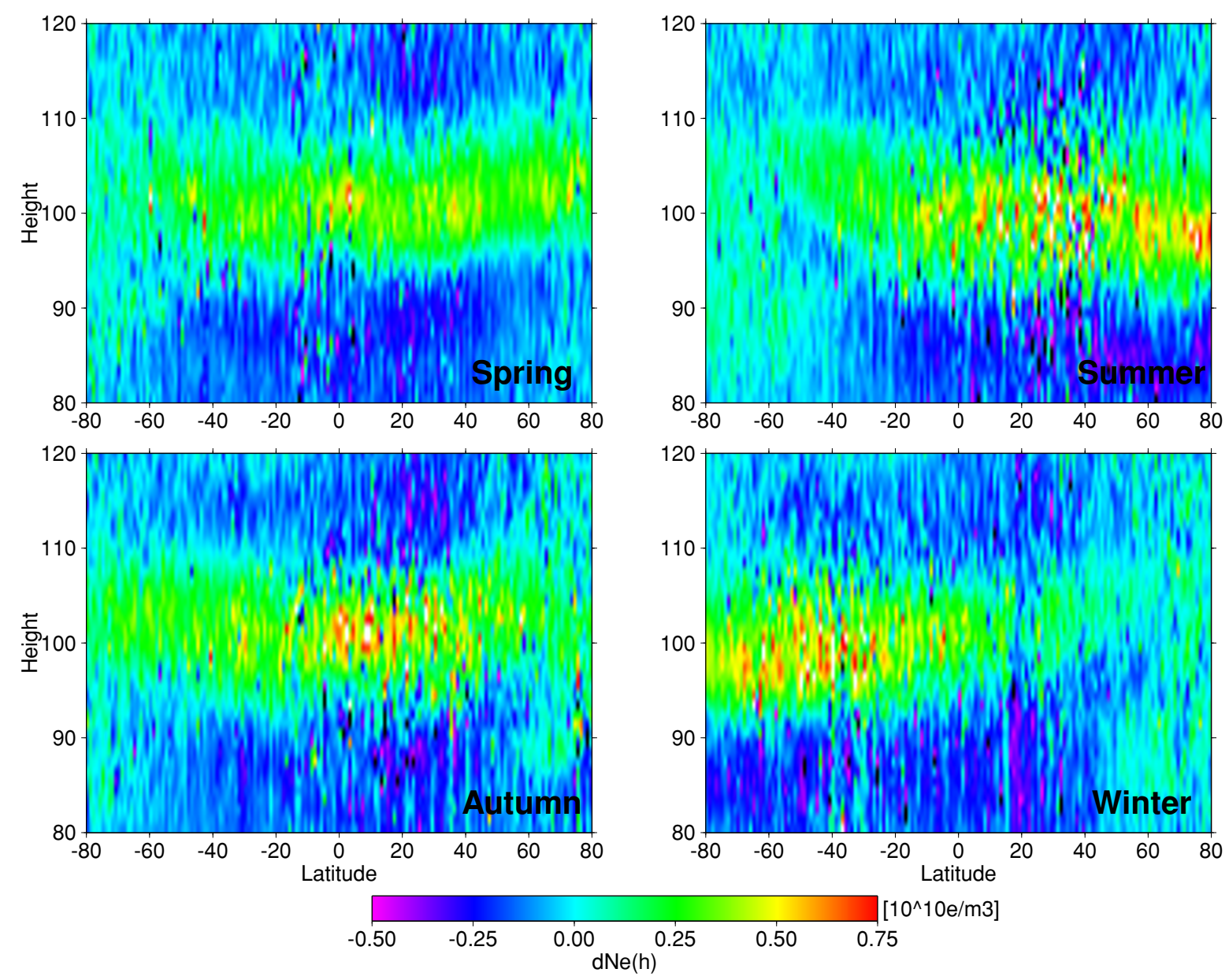

Fig. 3. Seasonal mean vertical profiles of ionospheric irregularities in the E layer against latitude during daytime period (5 LT to 22 LT). Spring, Summer, Autumn and Winter are upper-left, upper-right, lower-left and lower-right panels respectively.

sity or, equivalently, larger maximum values in the fluctuation profiles computed with the technqiue used in this work $\left(\max \left\{\Delta N_{e}(h)\right\}\right)$. Once the maximum of each fluctuation profile and its location has been searched and grouped according to season, the global and seasonal distribution can be plotted (see Fig. 2). The maps (which correspond to daytime observations, from 5 LT to 22 LT) show a strong geographic and seasonal dependency as stated by different studies based on ground observations. The enhancement of this fluctuations in the Auroral regions (above $+60^{\circ}$ or under $-60^{\circ}$ of latitude) can be clearly seen during all seasons. Note as well the high enhancements in the summer hemispheres, which were already seen during low solar activity period with the GPS/MET satellite (Hocke et al., 2001). Specially the enhancements in Europe and Asia during northern summer hemisphere and near South America and Oceania during southern summer hemisphere. During nighttime (from 22 LT to 5 LT), these fluctuations are less important, but they show the same behavior as the case of daytime. These behaviours were pointed out in Whitehead (1989) (and references therein) and Mathews (1998), with ground based instruments, and in Hocke and Tsuda (2001b) with the GPS/MET data, during low solar activity. With this work this behavior is also confirmed during high solar activity.

With the high resolution vertical profiles of irregularities obtained from the CHAMP GPS observations, it is possible as well to obtain a seasonal description of the irregularities in height versus latitude (see Fig. 3, for the daytime period). This figure is consistent with Fig. 2, showing that the ionospheric irregularities are mainly enhanced in summer hemisphere. Moreover it can be seen how the maximums are located around $100 \mathrm{~km}$. During nighttime (see Fig. 4) the daytime pattern disappears, nevertheless the plot shows a clear enhancement for all seasons in the high latitudinal bands, coinciding with the Auroral region. Changes of phase of 180 degrees in the fluctuation profiles, that could be masked in the averaging done to obtain Figs. 3 and 4, have not been detected in the data set of this work.

\section{Discussion}

With the occultation data from the CHAMP satellite, this work shows the global and seasonal distribution of the ionospheric irregularities of electron density in the E layer. In this context and based on the proof-of-concept carried out with the GPS/MET satellite, this new data set with CHAMP data confirms other results corresonding to previous LEO missions as well as with ground based instruments. In particular, the enhancement of irregularities in the auroral regions, in mid latitudes and summer hemispheres. Moreover, the data with CHAMP satellite shows that the spoardic E phenonema shows similar events in Solar Maximum condi- 

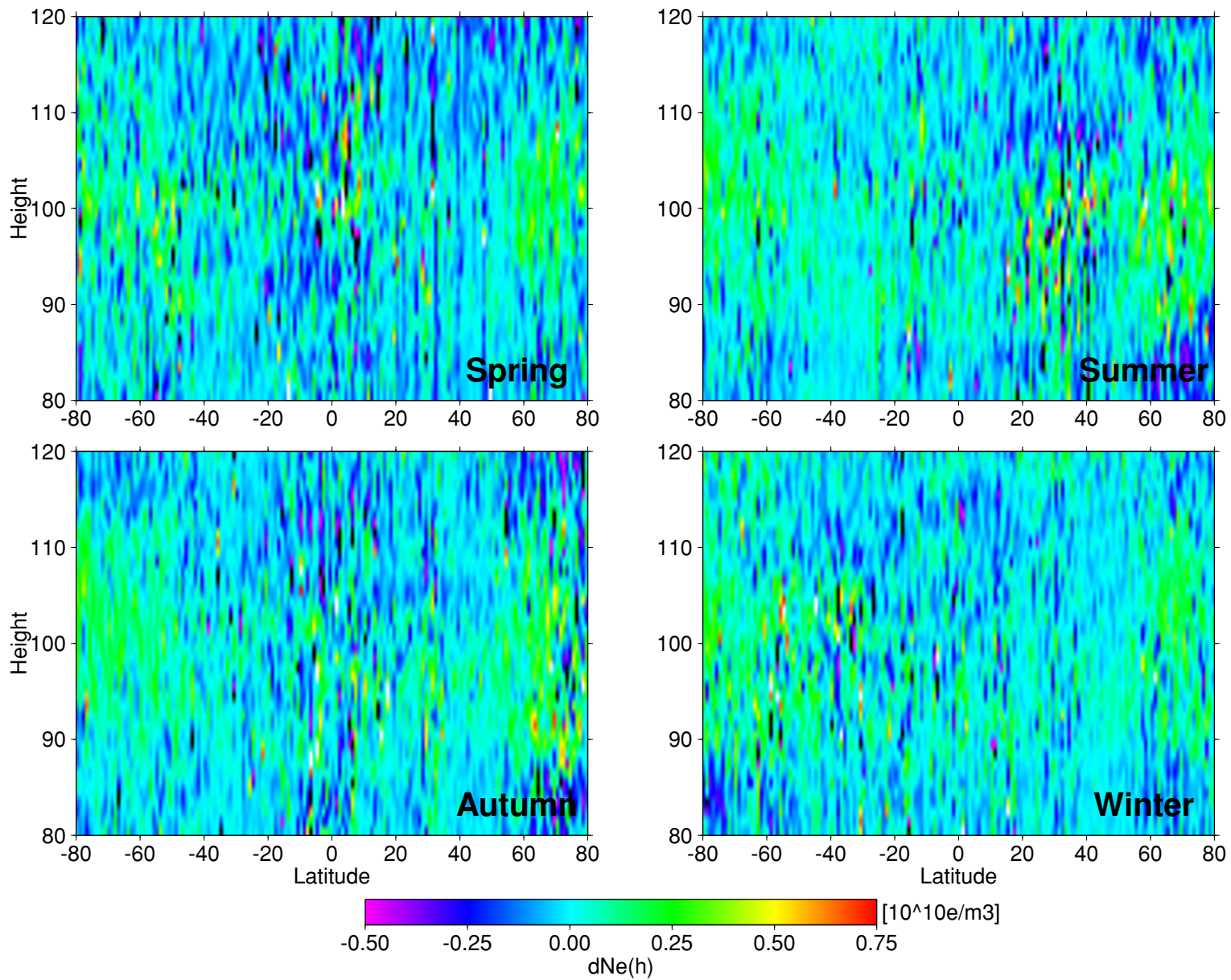

Fig. 4. Seasonal mean vertical profiles of ionospheric irregularities in the E layer against latitude during nighttime period (22 LT to 5 LT). Spring, Summer, Autumn and Winter are upper-left, upper-right, lower-left and lower-right panels respectively.

tions (corresponding to this data set) and Solar Minimum period (as shown with the results of GPS/MET in 1995/7). This type of high resolution vertical information of the ionospheric irregularities in conjunction with neutral atmospheric data may enhance the comprehension of the coupling processes that exist between these two atmospheric layers. In particular, the coupling of the neutral winds of the atmoshpere and the ionosphere through the wind-shear theory. Additionally, with the advent of new LEO missions such as COSMIC or EQUARS in the following years, the larger available data sets will provide with the opportunity to study not only the global or seasonal behavior of the sporadic $E$ layer as done in this work, but particular events over specific locations of the globe or different ionospheric or atmospheric conditions.

Acknowledgments. CHAMP data has been obtained from the GENESIS JPL ftp server. Plots have been plotted with the software package Generic Mapping Tools (GMT). This research has been fully supported by the Kyoto University Active Geosphere Investigations for the 21st Century COE (KAGI21), which was approved by the Ministry of Education, Culture, Sports, Science, and Technology (MEXT) of Japan.

\section{References}

Ahmad, B. and G. L. Tyler, The two-dimensional resolution kernel associated with retrieval of ionospheric and atmospheric refractivity profiles by Abelian inversion of radio occultation phase data, Radio Science,
33(1), 129-142, January-February 1998

Hajj, G. A. and L. J. Romans, Ionosphere electron density profiles obtained with the Global Positioning System: Results from the GPS/MET experiment, Radio Science, 33, 175-190, 1998.

Hardy, K. R., G. A. Hajj, E. R. Kursinski, and R. Ibanez-Meier, Accuracies of atmospheric profiles obtained from GPS occultations, Proceedings of the ION GPS-93 Conference, 1545-1556, 1993.

Hocke, K. and T. Tsuda, Gravity waves and Ionospheric irregularities over tropical convection zones observed by GPS/MET Radio Occultation, Geophys. Res. Lett., 28(14), 2815-2818, July 2001a.

Hocke, K. and T. Tsuda, Using GPS satellites to study plasma irregularities, GPS World, 34-36, July, 2001b.

Hocke, K., K. Igarashi, M. Nakamura, P. Wilkinson, J. Wu, A. Pavelyev, and J. Wickert, Global Sounding of sporadic E layers by the GPS/MET radio occultation experiment, J. Atmos. Solar-Terr. Phys., 63, 19731980, 2001.

Hocke, K. and K. Igarashi, Structure of the Earth's lower ionosphere observed by GPS/MET radio occultation, J. Geophys. Res., 107(A5), 2002.

Mathews, J. D., Sporadic E: current views and recent progress, J. Atmos Solar-Terr. Phys., 60, 413-435, 1998.

Schreiner, W. S., S. V. Sokolovskiy, C. Rocken, and D. C. Hunt, Analysis and validation of GPS/MET radio occultation data in the ionosphere, Radio Science, 34, 949-966, 1999.

Whitehead, J. D., The formation of the sporadic-E layer in the temperate zones, J. Atmos. Terr. Phys., 29, 49-58, 1961.

J. D. Whitehead, Recent work on mid-latitude and equatorial sporadic-E, J. Atmos. Terr. Phys., 51, 401-424, 1989.

M. Garcia-Fernandez (e-mail: mgacia@rish.kyoto-u.ac.jp) and T. Tsuda 